\title{
WebQuest e New WebQuest: per una didattica della storia in ambiente digitale
}

\author{
di Aldo Gianluigi Salassa
}

Giu 23, $2021 \mid \underline{\text { In evidenza, }}$ Pensare la didattica $\mid \underline{0 \mid}$

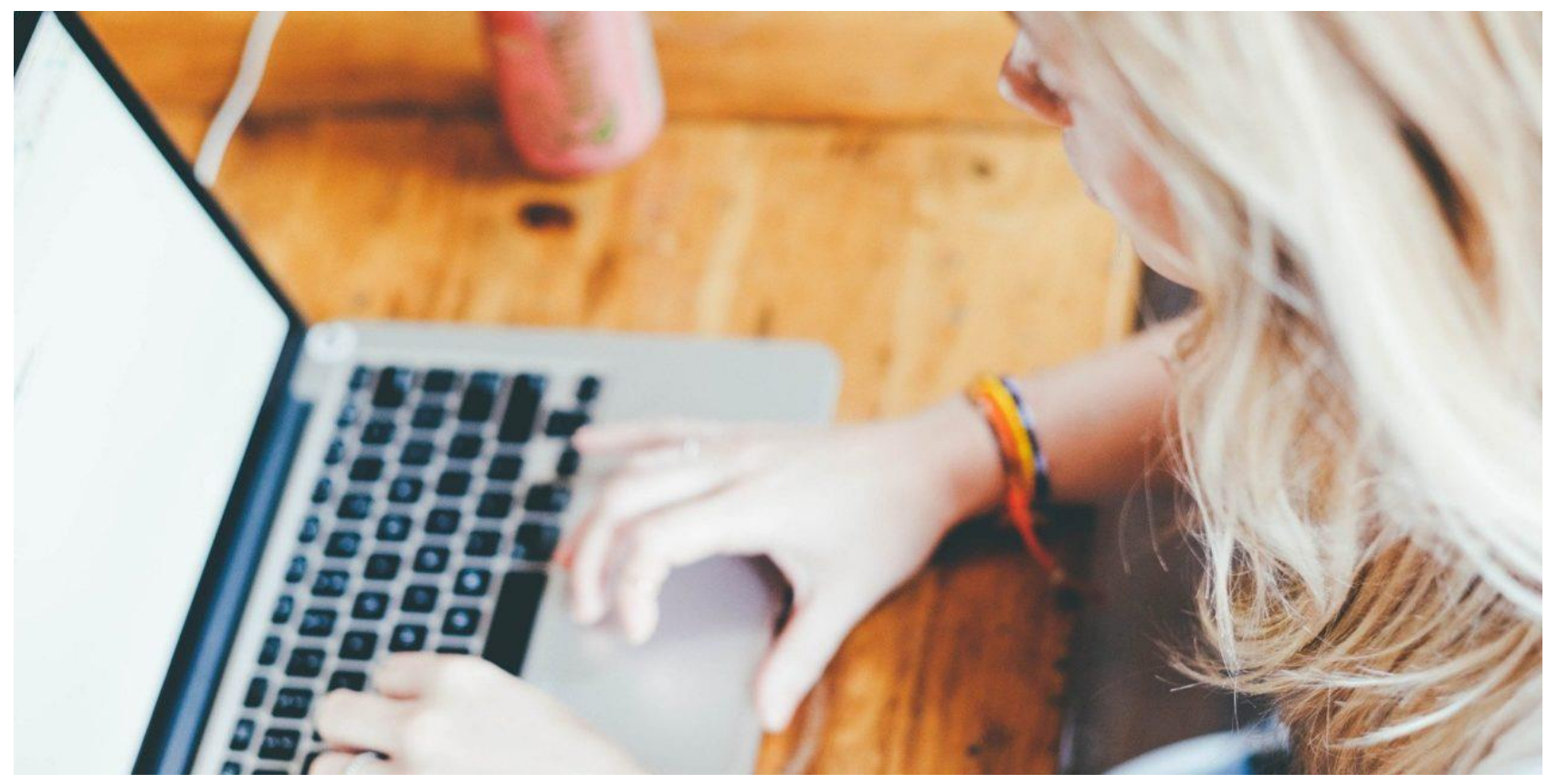

\begin{abstract}
Pratica molto in voga agli albori del web, il WebQuest ha perso progressivamente centralità anche in relazione alla rapida evoluzione di internet e al nascere del cosiddetto web 2.0. Le reti di utenti e le enciclopedie partecipativa hanno messo in crisi questa pratica, che tuttavia sta fiorendo a nuova vita negli ultimi anni, grazie soprattutto al lavoro di Bearzi, e Colazzo nel 2017. Ma com'è strutturato un WebQuest? Quali sono le sue potenzialità, soprattutto dopo un anno di scuola trascorso per lo più davanti agli schermi dei computer?
\end{abstract}

Premessa (una volta tanto) indispensabile

Sostiene Luciano Floridi, docente di Filosofia e Etica dell'informazione a Oxford, che siamo da tempo entrati in un nuovo mondo, quello onlife, ${ }^{, 1]}$ in cui la nostra vita risulta essere allo stesso tempo reale e virtuale, analogica e digitale, sulla terra e nel web.

Evidentemente, la scuola non può non risentire dei cambiamenti in corso. Può tentare - impresa disperata e di dubbia utilità - di opporvi resistenza; ma in nome di che cosa, poi? O può cercare di continuare a svolgere, anche nella nuova dimensione esistenziale dell' onlife, il suo compito di creare cittadini consapevoli attraverso l'acquisizione di un sapere critico.

La scelta sembra, in teoria, scontata ma in pratica non lo è. 
Ogni insegnante, infatti, nonostante le sperimentazioni didattiche cui ha cercato, nel tempo, di dare il suo contributo, quando inizia un nuovo anno scolastico è chiamato a porsi alcune domande fondamentali:

1. Sono davvero disposto a superare una certa tradizione educativa, ancora molto diffusa, basata sul binomio «lezione frontale + libro di testo»?

2. Sono davvero disposto a mettermi in gioco, ad abbandonare una visione trasmissiva dell'insegnamento e passiva dell'apprendimento, in vista di un'altra che preveda, per lo studente, un ruolo da protagonista nella costruzione del suo sapere e, per il docente, un ruolo di facilitatore, se non di vero e proprio designer, dell'azione didattica?

3. Sono davvero disposto, insegnando storia, a preoccuparmi di formare "una testa ben fatta piuttosto che una testa ben piena" (per citare Montaigne), ${ }^{[2]}$ abituando quindi lo studente a "pensare storicamente"? [3]

4. Sono, infine, davvero disposto a cambiare la mia modalità di insegnamento, sfruttando appieno le risorse digitali oggi disponibili e abituando i ragazzi a usare il web come ambiente di studio e apprendimento, e non solo come spazio virtuale per le relazioni sociali?

Se si risponde con quattro sì chiari e convinti a queste domande si può iniziare a ragionare su quali strumenti utilizzare per procedere. Qui ci si concentrerà su una metodologia che ha già una consolidata tradizione e che, se opportunamente aggiornata, mantiene la sua efficacia didattica per tutti gli ordini di scuola (Università compresa): il WebQuest (da qui in avanti WQ).

\section{Il WebQuest}

L'espressione WQ può essere genericamente tradotta con "indagine condotta sul web".

In pratica, nella sua forma classica, si tratta di una ricerca in rete assegnata alla classe ma guidata passo dopo passo dall'insegnante, al fine di evitare che gli studenti si affidino a una navigazione tanto spontanea quanto acritica e casuale.

Il WQ è una metodologia didattica il cui modello è stato formalizzato per la prima volta nel 1995 da Bernie Dodge (professore di tecnologia educativa all'università di San Diego, California) e sviluppato, per un uso scolastico, con la collaborazione di Tom March.

Dodge si era reso conto che il computer era ormai divenuto di uso comune e, pertanto, andava integrato in modo sistematico nell'insegnamento di tutte le discipline. Nel 2001, dopo qualche anno di sperimentazioni, Dodge ne ha dato una definizione precisa:

Attività orientata alla ricerca in cui tutte o la maggior parte delle informazioni utilizzate dagli studenti sono tratte dal web. I WQ sono programmati per ottimizzare i tempi di studio; per concentrarsi, piuttosto che sull'acquisizione, sull'utilizzo dell'informazione; per supportare le attività cognitive ai livelli di analisi, sintesi e valutazione. ${ }^{4]}$

Su questa definizione dovremo ritornare più avanti, perché ha il pregio di indicare con chiarezza quali siano gli obiettivi e, in controluce, i limiti di una metodologia i cui presupposti, nel caso si decida di adottarla, non devono essere fraintesi. 


\section{WebQuest: 6 step}

La struttura standard di WQ, condivisa a livello internazionale, è costituita da 6 fasi:

1. Introduzione (Introduction)

2. Compito (Task)

3. Risorse (Resources)

4. Processo (Process)

5. Valutazione (Evaluation)

6. Conclusione (Conclusion)

Occorre sottolineare subito che è fondamentale che l'insegnante, al momento della scelta dell'argomento e prima di organizzare il lavoro in classe, stabilisca tempi certi di svolgimento dell'attività.

A questo proposito, Dodge distingue tra WQ di breve e di lunga durata.

Nel primo caso, il WQ occuperà un arco di tempo limitato (due-tre lezioni), prevederà soprattutto l'acquisizione di - non troppo numerosi - dati conoscitivi e richiederà un prodotto finale semplice (testo di sintesi, mappa concettuale, schema).

Nel secondo caso, il WQ si baserà invece su un corpus più ampio e rappresentativo di fonti, necessiterà di un lavoro di gruppo e richiederà un livello maggiore di elaborazione, nonché la realizzazione di un prodotto finale più complesso.

Esaminiamo ora, una per una, le varie parti della struttura.

\section{Introduzione}

Si tratta di una presentazione che fornisce informazioni essenziali sull'argomento, spesso anticipando la domanda fondamentale che ispira l'intera indagine.

In un WQ dedicato al Movimento No Global, per esempio, ${ }^{[5]}$ l'introduzione potrebbe essere questa:

No global è un'espressione inglese divenuta di uso comune anche nella nostra lingua; significa "contrario, che si oppone alla globalizzazione".

$\grave{E}$ affine ad altre espressioni come, ad esempio, no logo ("contro le griffe", cioè contro tutto ciò che è "firmato"), non a caso il titolo di un libro scritto dalla giornalista e attivista canadese Naomi Klein (2000) ritenuto il manifesto dell'antiglobalizzazione. No global è generalmente accostato al sostantivo movimento con lo scopo di indicare una galassia di organizzazioni, gruppi e persone di tutto il mondo che combattono le politiche del capitalismo internazionale, rappresentato da istituzioni come il FMI (Fondo Monetario Internazionale) e la WTO (World Trade Organisation). I movimenti no global (o movimento dei movimenti, come spesso si sente dire) hanno in comune gli obiettivi esposti nel manifesto approvato al Social Forum di Porto Alegre (Brasile) nel 2002: annullamento del debito dei paesi poveri, abolizione dei paradisi fiscali, protezione dell'ambiente e della biodiversità, sostegno ai diritti dei lavoratori, alla parità tra uomo e donna, diffusione della democrazia nel mondo, condanna del terrorismo e della guerra.

Il principale slogan del movimento no global è: Un altro mondo è possibile.

Viceversa, se si assegna un WQ dal titolo Due crac finanziari a confronto: Firenze 1343/New York 2008, la presentazione dell'argomento potrebbe essere diversa: 
Oggetto della ricerca è l'accostamento, che può apparire ardito, tra un evento finanziario del Trecento - il crollo delle banche fiorentine dei Peruzzi e dei Bardi (1343-46) - e uno contemporaneo - ovvero il recente fallimento della banca americana Lehman Brothers (2008), manifestazione eclatante della gravissima crisi finanziaria internazionale che ha caratterizzato questi ultimi anni della nostra storia. In realtà, il paragone tra i due eventi si è imposto naturalmente, sin dal primo momento, agli osservatori; e nemmeno tanto tra gli storici, quanto tra gli analisti economici che vi hanno scorto utile materia per una riflessione sui meccanismi ricorrenti delle crisi finanziarie.

Il crac del XIV secolo fu causato dall'insolvenza del monarca inglese Edoardo III verso le banche fiorentine che gli avevano prestato una cifra colossale per l'epoca (circa un milione e trecentocinquantacinquemila fiorini d'oro) per sostenere le sue spese di guerra.

Oltre sei secoli e mezzo dopo, la Lehman Brothers, una delle più antiche banche americane (fondata nel 1850 in Alabama), fra le maggiori degli Stati Uniti, si è trovata anch'essa di fronte ad un passivo record (613 miliardi di dollari), causato questa volta non dall'insolvenza di un singolo debitore, come il monarca inglese, ma dalla crisi dei mutui subprime, ovvero i mutui per la casa concessi, con troppa leggerezza, a migliaia e migliaia di persone di scarsa affidabilità economica allo scopo di ricavare elevati tassi di interesse. Un fallimento, quello della Lehman Brothers, che ha cambiato in modo forse irreversibile il volto della finanza mondiale.

In questo caso la presentazione non punta tanto a descrivere i due eventi, sui quali gli studenti saranno successivamente chiamati a raccogliere informazioni, ma a spiegare la ragione di un accostamento che si regge su forti analogie rilevate prima di tutto dagli economisti, sempre alla ricerca di modelli generali di interpretazione delle congiunture economiche.

\section{Compito}

È la parte più importante del WQ. Indica sia quale debba essere il prodotto finale, oggi si direbbe compito autentico (un articolo di giornale, una relazione, un discorso da tenere in pubblico, un saggio breve, un artefatto multimediale), ${ }^{[6]}$ sia quale registro comunicativo e quale attitudine cognitiva e metacognitiva vengano richiesti allo studente.

Riguardo al secondo aspetto, Lodge ha proposto a suo tempo un'articolata tassonomia dei compiti da assegnare agli studenti, che può costituire ancora oggi una guida per i docenti che si accingono a progettare un WQ.

Semplificando e rielaborando la classificazione di Lodge, anche alla luce delle esigenze specifiche della didattica della storia, si possono individuare alcune fra le possibili tipologie di attività:

- compiti ripetitivo-compilativi: raccogliere e riassumere informazioni, documentare;

- compiti analitico-interpretativi: rielaborare e confrontare informazioni; comparare fenomeni e processi, rappresentare graficamente dati, ecc.;

- compiti giornalistici: scrivere un articolo; effettuare un'intervista reale, virtuale oppure immaginaria (per esempio a un personaggio storico del passato), ecc.;

- compiti indagativi: analizzare, risolvere un caso, ricostruire un evento;

- compiti argomentativi: a partire da una questione storica controversa, assumere un punto di vista e proporre una narrazione e/o un'interpretazione convincente.

Le varie tipologie, com'è ovvio, non si escludono rigidamente, ma possono intrecciarsi e coesistere in uno stesso percorso, anche se, di solito, è una a prevalere nella consegna. 
Il primo compito indicato (di tipo informativo) è il più semplice.

Ad esempio, se si volesse proporre un WQ sull'incidente di Chernobyl (26 aprile 1986: una delle più grandi catastrofi ambientali del secondo Novecento) si potrebbe chiedere agli studenti di esaminare diverse fonti, soprattutto audiovisive, e chiedere - compito relativamente semplice - la stesura di una relazione in forma multimediale da esporre ai compagni di classe. ${ }^{[7]}$

Più complesse, invece, altre tipologie di compiti.

Per fare un esempio di alto livello di difficoltà, si potrebbe proporre un WQ incentrato sulla presentazione di un' importante disciplina "ausiliaria" della storia: la demografia. Il compito assegnato agli studenti, in questo caso, potrebbe essere il reperimento di fonti (dati demografici Istat, Eurostat, ONU e così via) in vista dell'analisi di una delicata questione di carattere storicosociale: l'invecchiamento della popolazione (italiana o europea) con i problemi che ne derivano. ${ }^{[8]}$

Particolarmente motivanti e gradite agli studenti sono, di solito, le ricerche che chiedono di ricostruire una vicenda storica dai risvolti poco chiari, se non addirittura assimilabili al giallo o al mistery. Emblematico, a questo proposito, potrebbe essere un WQ dedicato alla Congiura dei $P a z z i,[9]$ dal nome della potente famiglia fiorentina che cercò di abbattere la signoria medicea a Firenze con un complotto, culminato il 26 aprile 1478 nell'attentato in Cattedrale contro Giuliano e Lorenzo de Medici (il primo viene assassinato, mentre il secondo riesce a salvarsi attuando poi una feroce vendetta contro i cospiratori).

La Congiura dei Pazzi può essere davvero assimilata a un giallo; anzi, a un intrigo internazionale, nel quale si trovano coinvolte le principali figure politiche dell'Italia (e in parte anche dell'Europa) dell'epoca. Agli studenti si potrebbe chiedere di simulare un'indagine poliziesca, che preveda una meticolosa ricostruzione dei fatti attraverso varie operazioni:

1. esame delle testimonianze (documenti, racconti);

2. esame medico (virtuale!) del corpo e dei relativi organi della vittima (autopsia);

3. esame della "scena del crimine" alla ricerca di indizi;

4. identificazione degli esecutori materiali dell'attentato;

5. individuazione dei possibili moventi; identificazione del mandante (o dei mandanti) del delitto.

\section{Risorse}

Si tratta di uno step fondamentale, in cui l'insegnante fornisce agli studenti le informazioni necessarie ad avviare, sviluppare e/o problematizzare l'argomento.

Come scrive Lodge nella definizione del 2001, i WQ «sono programmati per [...] concentrarsi, piuttosto che sull'acquisizione, sull'utilizzo dell'informazione». $\frac{[10]}{[}$

Si tratta un'indicazione precisa, che invita il docente a farsi carico della selezione preliminare delle fonti, scegliendo quelle che più gli sembrano adeguate al compito e che, possibilmente, orientino e non disorientino (!) lo studente. Questa è la base di partenza, anche se, per le classi terminali della Secondaria superiore, si può prevedere che lo studente integri in parte, con una ricerca libera, le proposte dell'insegnante. 
Le fonti considerate potranno essere scritte, visive e audiovisive e verranno per la maggior parte ricavate dal web (link): in caso contrario, com'è ovvio, la ricerca non avrebbe le caratteristiche di un WQ, né costituirebbe una palestra di source criticism digitale.

Le fonti, inoltre, dovranno essere presentate in maniera ragionata e organizzata secondo criteri di gradualità.

In via preliminare, ad esempio, si potrà proporre la lettura di buone voci enciclopediche (Wikipedia, anche quella britannica, Treccani, compresa quella per i ragazzi, e così via), magari integrate da repertori di concetti e/o parole chiave per riprendere nozioni fondamentali. In seguito, si passerà a proporre link di approfondimento, commisurati al grado di difficoltà e complessità del percorso scelto. $\stackrel{111}{ }$

La necessità di selezionare attentamente le fonti obbliga di fatto il docente a impegnarsi in un'attenta analisi delle informazioni ricavate dalla rete, per verificarne la qualità e l'attendibilità (validazione).

\section{Processo}

In questa sezione, l'insegnante è chiamato a suggerire la procedura di lavoro, elencando le attività e le operazioni che gli studenti devono svolgere per portare a termine la ricerca, compresa la divisione dei compiti e la ripartizione dei materiali. In determinati casi, il docente può decidere di agevolare il compito fornendo una serie di aiuti (elenchi di domande-guida, schemi, scalette, mappe concettuali e così via). Va comunque ribadito che il WQ, pur prevedendo un ruolo attivo e orientativo dell'insegnante, resta un'attività di problem solving (PS). ${ }^{[12]}$

Se già non lo si è fatto al momento dell'illustrazione del compito, può essere questo il momento in cui chiedere allo studente di simulare una situazione reale (mettersi nei panni di...), allo scopo di stimolare la sua immaginazione e fornirgli una bussola per meglio orientarsi tra le fonti.

Nel caso del WQ, già presentato, sui Due Crac a confronto, si può proporre, ad esempio, che la classe si divida in tre gruppi, ciascuno dei quali studierà i due fatti in chiave, rispettivamente, giornalistica, storica ed economica. Ogni gruppo esprimerà poi un portavoce. I tre studenti selezionati dovranno simulare, di fronte alla classe, un dibattito televisivo (un talk) cui partecipano un giornalista-moderatore, uno storico e un economista. Al primo spetterà di porre domande ai due esperti, chiedere chiarimenti sulle loro risposte, tirare le conclusioni del dibattito; al secondo, di spiegare le ragioni del crac delle banche fiorentine; al terzo, di descrivere la vicenda del fallimento Lehman Brothers avanzando un paragone con quelli dei Peruzzi e dei Bardi.

\section{Valutazione}

Non essendo un'attività didattica tradizionale, il WQ esige che si dedichi una particolare attenzione al momento della valutazione. Meglio ricorrere a una griglia: se ne possono trovare di utili e affidabili in diverse pubblicazioni didattiche $\mathrm{o}$ in rete. $\frac{[13]}{}$

\section{Conclusione}

È il momento in cui l'insegnante chiede agli studenti di riepilogare le principali tappe del percorso (debriefing) e di elaborare il significato dell'attività svolta (momento metacognitivo). 
Anche in questo caso, può essere opportuno predisporre una scheda, compilando la quale gli studenti potranno ricostruire i vari momenti del percorso, evidenziare positività e criticità riscontrate nel lavoro svolto (singolarmente e in gruppo), mettere a confronto obiettivi di partenza e risultati raggiunti e così via.

\section{Oggi, un New Webquest}

In Italia la metodologia del WQ non ha avuto, nel recente passato, troppa fortuna presso gli insegnanti. Le ragioni di questo fatto sono molteplici, ma due soprattutto meritano di essere segnalate: il ritardo cronico della scuola italiana nell' adottare e integrare le ITC nella didattica; la diffidenza di molti docenti nei confronti del digitale come ambiente di apprendimento (in pratica, si tratta probabilmente delle due facce di una stessa medaglia).

Notevolissima, invece, la diffusione del WQ in Spagna e nei Paesi di lingua spagnola, soprattutto nel mondo universitario.

Diffusione a parte, nel secondo decennio degli anni 2000 il WQ è parso avviarsi a un inesorabile declino. L'avvento del Web 2.0, infatti, ha assestato un duro colpo a una metodologia nata e sviluppata in una fase in cui il Word Wide Web stava iniziando ad affermarsi e, ancora, non permetteva l'interattività. Inoltre, se torniamo per un'ultima volta sulla definizione di Bernie Dodge proposta all'inizio, i WQ "classici" sembrano essere condizionati da una sorta di "impronta genetica": quella di essere «programmati per ottimizzare i tempi di studio». Con questa delimitazione di orizzonti, il WQ classico rischia di risultare uno strumento inadeguato a interagire con le didattiche costruttivistiche e partecipative che sono, oggi, ritenute indispensabili dai pedagogisti per migliorare i processi di apprendimento.

In un loro recente saggio, però, Bearzi e Colazzo ${ }^{[14]}$ rilanciano il WQ come una proposta forte, non solo didattica ma anche educativa, in grado - scrive Piergiuseppe Ellerani nella Presentazione al volume - «di interpretare 'che cosa fare in classe' per formare competenze, per interpretare l'insegnamento come azione creativa, motivante e contestualmente attenta alla formazione del curricolo, per alimentare capacità di scoperta e di ricerca nella soluzione di problemi reali sociali, culturali e territoriali. E soprattutto, in grado di connettere gli universi di senso e di conoscenza formali e informali, 'fuori' e 'dentro' la classe». $\stackrel{[15]}{ }$

Un WQ aperto all'implementazione di metodologie didattiche innovative si prefigge (sintetizzando e parafrasando Bearzi e Colazzo) i seguenti obiettivi:

- educare i giovani a un uso critico e responsabile del web trasformando la rete in un'opportunità di apprendimento;

- creare un ambiente formativo in cui lo studente possa apprendere in modo non trasmissivo ma per scoperta, costruendo la propria conoscenza;

- sviluppare negli studenti la capacità di lavorare in gruppo trasformando la classe in comunità di apprendimento;

- realizzare un'attività che ponga al centro lo studente (student centered learning) e attribuisca al docente - visto come tutor/facilitatore - determinate azioni ovvero: la definizione di un compito autentico; l'organizzazione cooperativa dell'apprendimento (cooperative learning) tramite la metodologia del laboratorio attivo (learning by doing); l'indicazione degli strumenti e delle risorse da utilizzare; l'individuazione dei luoghi di 
incontro (anche esterni: ambiente formativo complex); il monitoraggio e la co-revisione dei prodotti, con relativa covalutazione.

Per concludere richiamiamo la definizione aggiornata di New WebQuest che Bearzi e Colazzo propongono nel loro saggio:

Nwq è un'attività creativa cooperativa, implicante approfondimento e ricerca, che valorizza in un ambiente di apprendimento blended le opportunità formative offerte da contenuti e applicazioni presenti nel web. Co-creando un prodotto più o meno ampiamente condivisibile, comunità di apprendimento, formate da un gruppo dei pari e da un docente facilitatore, sviluppano motivazione intrinseca all'apprendimento, affinando funzioni cognitive e metacognitive, attitudine al pensiero critico, creativo, divergente e laterale, al problem solving e al pensiero indiziario e abduttivo, capacità relazionali e 'cosmopolitiche'.116]

Note:

[1] Per un primo accostamento al concetto di onlife e al relativo paragone con la "società delle mangrovie", vedi un'intervista con il filosofo in https://www.repubblica.it/dossier/tecnologia/onlife/2019/09/29/news/repubblica_onlife_luciano_ floridi-237286128/.

Sull'interrelazione del concetto di onlife con quelli di infosfera e iperstoria, vedi https://corriereinnovazione.corriere.it/2020/06/25/floridi-siamo-entrati-una-nuova-epoca-oggiviviamo-nell-iperstoria-f470575c-b6f8-11ea-877f-fd70c3a48db9.shtml.

[2] il celebre aforisma si trova nel primo libro dei Saggi, nel capitolo Sull'Istruzione dei fanciulli, che è successivo a quello Sulla pedanteria. Enunciando così la principale caratteristica del precettore ideale, Montaigne si schiera, per dirla in modo attuale, contro il nozionismo e a favore delle competenze.

[3] È intorno al Reading like a historian, e al suo valore pedagogico-educativo per una cittadinanza consapevole, che ruota la riflessione di Sam Wineburg, docente di storia e didattica della storia alla Stanford University in California. Cfr. l'articolo di Paolo Ceccoli:

http://www.novecento.org/pensare-la-didattica/pensare-come-uno-storico-non-e-naturale-3402/.

[4] Cfr. FOCUS. Five rules for writing a great webquest, «Learning and Leading with Technology» 28-8, pp. 6-9 e 58, citato e tradotto in Francesco Bearzi, Salvatore Colazzo, New WebQuest. Apprendimento cooperativo, comunità creative di ricerca e complex learning nella scuola di oggi, Franco Angeli, Milano 2017.

[5] Tutti i WQ e i relativi testi che, da questo momento in poi, verranno proposti nell'articolo sono reperibili nella versione digitale del manuale di Pasquale Armocida, Aldo Gianluigi Salassa, Storialink, Edizioni Scolastiche Bruno Mondadori, Milano 2013, destinato agli istituti secondari superiori.

[6] Gli artefatti multimediali che gli studenti oggi prediligono sono quelli di forma narrativa: video, storytelling, Instagram stories. 
[7] Armocida, Salassa, 2013.

[8] Armocida, Salassa, 2013.

[9] Armocida, Salassa, 2013.

[10] Armocida, Salassa, 2013.

$111]$ Tra le fonti secondarie dovrebbero trovare naturalmente posto anche quelle narrative (opere letterarie, filmiche, fiction televisive, ecc.) che, adeguatamente inquadrate e contestualizzate, consentono di avviare interessanti percorsi didattici.

In considerazione, poi, dell'importanza che sono venute assumendo di recente nella fruizione televisiva e in streaming, un posto particolare potrebbero avere, anche indipendentemente dalla loro qualità, le produzioni seriali di argomento storico. Per restare al caso appena citato della Congiura dei Pazzi, una fiction come quella «I Medici» - che tra l'altro dedica un episodio, il $7^{\circ}$ della Seconda stagione, all'evento del 1478 - sarebbe un'utile palestra per esercitazioni didattiche al crocevia tra conoscenza del passato, media literacy e uso pubblico della storia.

${ }^{[12]}$ È appena il caso di ricordare ciò che distingue il comune processo di apprendimento dal problem solving: il primo si basa su procedure innate o già acquisite e sulla comparazione di un problema con un altro già noto; il PS, invece, presuppone un ragionamento strutturato, che si serve di determinate strategie e abilità per risolvere una situazione inedita e complessa.

[13] Una griglia molto articolata si può trovare in Pasquale Armocida, Aldo Gianluigi Salassa, Storialink. Guida per l'insegnante, Edizioni scolastiche Bruno Mondadori, Milano 2012, p. 47

${ }^{[14]}$ Francesco Bearzi è insegnante di liceo e Ph.D. presso l'Università La Sapienza di Roma; Salvatore Colazzo è professore ordinario di Pedagogia sperimentale all'Università del Salento. Il libro cui si fa riferimento è Bearzi, Colazzo, New WebQuest, 2017.

${ }^{[15]}$ Bearzi, Colazzo, 2017, p. 621

[16] Bearzi, Colazzo, 2017, p. 2943 e ss. dell' edizione digitale.

Il volume propone interessanti esempi di New WebQuest -realizzati in varie scuole, pensati per diverse discipline e in certi casi multidisciplinari- distinguendo inoltre fra NWQ di durata lunga (Long Term), almeno uno o due mesi, da altri più agili (Short o Medium Term), rispettivamente di 1-2 settimane e 3-6 settimane. Ovviamente si tratta di tempi comprensivi dell'attività che gli studenti svolgono in spazi e momenti extrascolastici: le ore di lezione impiegate dai docenti, infatti, sono state assai limitate. 